\title{
Deciphering the sounds of mice in social contexts
}

Sangiamo, D.T., Warren, M.R., and Neunuebel, J.P. Nat Neurosci 23, 411-422. https://doi.org/10.1038/s41593-020-0584-z (2020)

When Josh Neunuebel, a neuroscientist at the University of Delaware, saw the first plots his undergraduate student Dan Sangiamo had put together, his jaw dropped. The pattern was so striking that he didn't believe it was actually real. "We tried so many ways to break it," he recalls, "And it just kept holding up."

The plots were of male mouse ultrasonic vocalizations (USVs), recorded from groups of males and females behaving naturally. Published recently in Nature Neuroscience, it's a new look at the 'silent' language of mice that captures context that has been missing in prior studies of mouse vocalization: social interactions.

Mice 'talk' in a language that humans just can't hear. Their ultrasonic vocalization range spans 35 to $110 \mathrm{KHz}$, whereas our auditory system maxes out around $20 \mathrm{kHz}$. Prior work has suggested that there are different categories of mouse USVs, and that developmental stage and genetic differences can influence the way mice 'speak.' But it has been difficult to capture the social behaviors associated with those USVs.

"There were a lot of assumptions made about the vocalizations," says Neunuebel, "The problem was no one really knew who was vocalizing in social settings, so it made interpreting the role and function of these vocalizations really challenging."

So Neunuebel and his lab built a custom recording set up, consisting of a nylon mesh cage with eight microphones positioned around it. Adult black B6.CAST-Cdh23Ahl+/ Kjn mice-chosen because mice of this strain don't lose their hearing as they agewere marked with blond hair dye in unique patterns and placed in groups of two males and two females in the cage. In addition to the audio recording, the quartets were recorded visually and their activity tracked with a computer program called Motr.

The multi-microphone array allowed the team, via a computer program they coded, to triangulate the source of a sound

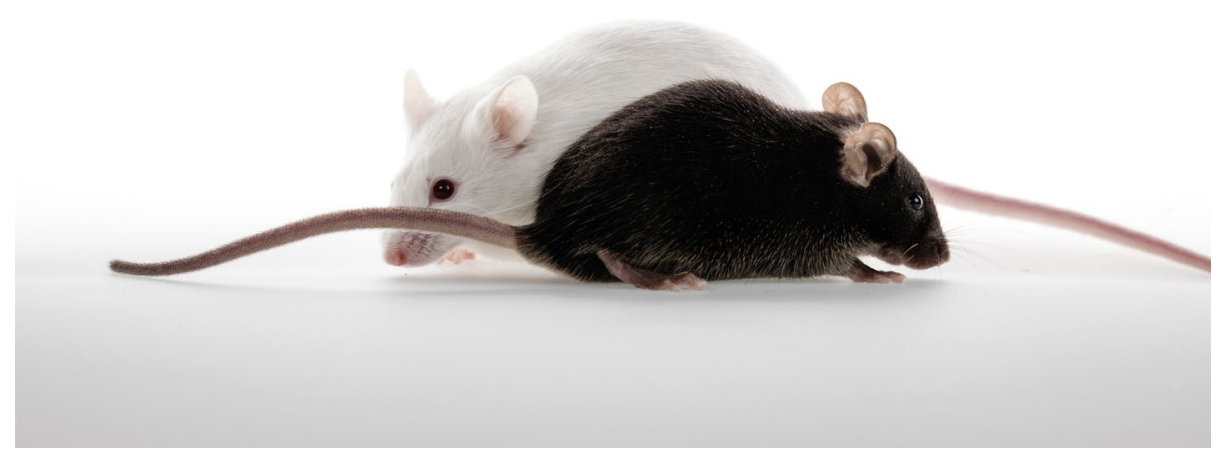

Secret no more? Research is underway to crack the ultrasonic language of naturally behaving mice. Credit: Georgejason / iStock / Getty Images Plus

to a particular mouse as the animals moved around the cage. "That was the first hurdle we had to overcome," says Neunuebel. With vocalizations assigned, the next big question was to determine whether there was any relationship between what the mice were saying and what they were doing.

Eleven groups of mice were recorded in five-hour sessions (after which the temptation to chew through the nylon would grow too great, says Neunuebel), and their actions were then classified into 8 categories with the Janelia Automatic Animal Behavior Annotator (JAABA), a machine learning algorithm. The categories were based on common mouse behaviors noted in the literature: walking; mutual circling; fighting; male chasing female; fleeing; fleeing from; male chase male; and male being chased.

It was then back to the supercomputer at the Univeristy of Delaware to process the combined auditory and visual dataover 100,000 vocal assignments and over 30,000 different behaviors. From all the data, considered individually and pooled together across the males, the lab was able to identify twenty-two distinct types of vocal signals and to then make correlations to different behaviors. From the data, they saw distinct increases in the pitch of male mice attempting to avoid another, while that of males exhibiting dominant behaviors, such as whether fighting or pursuing a female-rose.

"We have the precision now to follow what animals are saying and their specific behaviors," Neunuebel says, "And we know who's vocalizing.... The pieces are starting to come into a place now where the story is becoming much clearer."

Much work is to follow. For the current publication they focused on the male mice, as they produce over $80 \%$ of all USVs. "It was low hanging fruit," says Neunuebel. Females though will feature in future publications, and his lab is also using the recording set-up and their software to study how communication varies in different strains of mice, including mouse models of autism.

For others interested in deciphering their own animals, Neunuebel notes that the recording rig takes some money and time to build and there is a learning curve to the software, but that he's happy to share code and help with the technical details.

\section{Ellen P. Neff}

Published online: 16 March 2020

https://doi.org/10.1038/s41684-020-0524-2 